DOI: $10.15393 /$ j3.art.2017.3750

UDC 517.52

Vandanjay Adiyasuren, Tserendorj Batbold

\title{
EXTENSION OF THE REFINED GIBBS' INEQUALITY
}

\begin{abstract}
In this note, we give an extension of the refined Gibbs' inequality containing arithmetic and geometric means. As an application, we obtain converse and refinement of the arithmetic-geometric mean inequality.
\end{abstract}

Key words: arithmetic-geometric mean inequality, Jensen's inequality, log-function, Gibbs' inequality

2010 Mathematical Subject Classification: 26D15, 94A15

1. Introduction. Let $n \geq 2$ and $w_{1}, \ldots, w_{n}$ be non-negative real numbers such that $\sum_{j=1}^{n} w_{j}=1$. Let $A_{n}$ and $G_{n}$ denote the weighted arithmetic and geometric means of the positive real numbers $x_{1}, \ldots, x_{n}$, that is,

$$
A_{n}=\sum_{j=1}^{n} w_{j} x_{j} \quad \text { and } \quad G_{n}=\prod_{j=1}^{n} x_{j}^{w_{j}} .
$$

The arithmetic-geometric mean inequality asserts that

$$
A_{n} \geq G_{n} .
$$

For more details about the arithmetic-geometric mean inequality the reader is referred to [3]- 8 .

It is interesting that some classical inequalities such as arithmeticgeometric mean inequality (see [9]), the Jensen inequality (see [10]), the Hölder inequality (see [11]) play an important role in information sciences.

Let $p_{j}, q_{j}>0(j=1, \ldots, n)$ and $\sum_{j=1}^{n} p_{j}=\sum_{j=1}^{n} q_{j}$. Then,

$$
0 \leq \sum_{j=1}^{n} p_{j} \log \frac{p_{j}}{q_{j}}
$$


with equality if and only if $p_{j}=q_{j}(j=1, \ldots, n)$. This inequality is known in literature as the Gibbs' inequality (see [8, p. 382]). The Gibbs' inequality has many applications in information theory and also in mathematical statistics.

In 2004 Halliwell and Mercer [6] presented the following refinement of the Gibbs inequality:

Theorem 1. Let $p_{j}, q_{j}(j=1, \ldots, n)$ be positive real numbers satisfying $\sum_{j=1}^{n} p_{j}=\sum_{j=1}^{n} q_{j}$. Then,

$$
\sum_{j=1}^{n} \frac{q_{j}\left(q_{j}-p_{j}\right)^{2}}{q_{j}^{2}+\hat{M}_{j}} \leq \sum_{j=1}^{n} p_{j} \log \frac{p_{j}}{q_{j}} \leq \sum_{j=1}^{n} \frac{q_{j}\left(q_{j}-p_{j}\right)^{2}}{q_{j}^{2}+\hat{m}_{j}}
$$

where $\hat{m}_{j}=\min \left(p_{j}^{2}, q_{j}^{2}\right), \hat{M}_{j}=\max \left(p_{j}^{2}, q_{j}^{2}\right) \quad(j=1, \ldots, n)$.

In $2014 \mathrm{H}$. Alzer [2] proved the following refinement of (1):

Theorem 2. Let $\alpha, \beta \in \mathbb{R}$. Then, inequalities

$$
\sum_{j=1}^{n} \frac{q_{j}\left(q_{j}-p_{j}\right)^{2}}{q_{j}^{2}+\hat{m}_{j}^{\alpha} \hat{M}_{j}^{1-\alpha}} \leq \sum_{j=1}^{n} p_{j} \log \frac{p_{j}}{q_{j}} \leq \sum_{j=1}^{n} \frac{q_{j}\left(q_{j}-p_{j}\right)^{2}}{q_{j}^{2}+\hat{m}_{j}^{\beta} \hat{M}_{j}^{1-\beta}}
$$

hold for positive real numbers $p_{j}, q_{j}(j=1, \ldots, n)$ with $\sum_{j=1}^{n} p_{j}=\sum_{j=1}^{n} q_{j}$ if and only if $\alpha \leq 1 / 3$ and $\beta \geq 2 / 3$.

In this note we give an extension of (2) containing arithmetic and geometric means. As an application, we obtain refinement of the left-hand inequality in (1) and we also give a converse of the arithmetic-geometric mean inequality.

2. Main results. In order to prove our main results, we need the following lemmas.

Lemma 1. (see [2]) (i) If $0<x \leq 1$, then

$$
x-1-\frac{(x-1)^{2}}{x+x^{1 / 3}} \leq \log x \leq x-1-\frac{(x-1)^{2}}{x+1}
$$

with equality if and only if $x=1$.

(ii) If $x>1$, then

$$
x-1-\frac{(x-1)^{2}}{x+1}<\log x<x-1-\frac{(x-1)^{2}}{x+x^{1 / 3}} .
$$


Lemma 2. Let $f_{a}(x):=\frac{x(x-a)^{2}}{a\left(x^{2}+\max \left\{x^{2}, a^{2}\right\}\right)}+\log x, a>0$. Then $f_{a}$ is a concave function on $(0,+\infty)$.

Proof. If $x \geq a$, then $f_{a}(x)=\frac{(x-a)^{2}}{2 a x}+\log x$, and consequently,

$$
f_{a}^{\prime \prime}(x)=\frac{a-x}{x^{3}} \leq 0 .
$$

On the other hand, if $0<x<a$, then $f_{a}(x)=\frac{x(x-a)^{2}}{a\left(x^{2}+a^{2}\right)}+\log x$, which yields

$$
f_{a}^{\prime \prime}(x)=-\frac{a^{6}+7 a^{4} x^{2}-9 a^{2} x^{4}+x^{6}}{x^{2}\left(x^{2}+a^{2}\right)^{3}}=-\frac{\left(a^{2}-x^{2}\right)\left(a^{4}+8 a^{2} x^{2}-x^{4}\right)}{x^{2}\left(x^{2}+a^{2}\right)^{3}}<0 .
$$

Therefore, the function $f_{a}(x)$ is concave for $x>0$.

Lemma 3. (see [1]) Let $f_{a}$ be as defined in Lemma 2, $k \in\{2, \ldots, n-1\}$, and

$$
s_{k}:=\max _{1 \leq \mu_{1}<\cdots<\mu_{k} \leq n}\left[\left(\sum_{j=1}^{k} w_{\mu_{j}}\right) f_{A_{n}}\left(\frac{\sum_{j=1}^{k} w_{\mu_{j}} x_{\mu_{j}}}{\sum_{j=1}^{k} w_{\mu_{j}}}\right)-\sum_{j=1}^{k} w_{\mu_{j}} f_{A_{n}}\left(x_{\mu_{j}}\right)\right] .
$$

Then,

$$
0 \leq s_{2} \leq s_{3} \leq \cdots \leq s_{n-1} .
$$

First, we give an extension of (2) based on the corresponding result in [2].

Theorem 3. Let $\alpha, \beta \in \mathbb{R}$ and $m_{j}=\min \left(x_{j}^{2}, A_{n}^{2}\right), M_{j}=\max \left(x_{j}^{2}, A_{n}^{2}\right)$ $(j=1, \ldots, n)$. Then inequalities

$$
\frac{1}{A_{n}} \sum_{j=1}^{n} \frac{w_{j} x_{j}\left(x_{j}-A_{n}\right)^{2}}{x_{j}^{2}+m_{j}^{\alpha} M_{j}^{1-\alpha}} \leq \log A_{n}-\log G_{n} \leq \frac{1}{A_{n}} \sum_{j=1}^{n} \frac{w_{j} x_{j}\left(x_{j}-A_{n}\right)^{2}}{x_{j}^{2}+m_{j}^{\beta} M_{j}^{1-\beta}}
$$

hold if and only if $\alpha \leq 1 / 3$ and $\beta \geq 2 / 3$.

Proof. We follow the method of proof given in [2].

(Necessity) Since the sums on the left-hand side and on the right-hand side of (5) are increasing with respect to $\alpha$ and $\beta$, respectively, it suffices 
to prove (5) for $\alpha=1 / 3$ and $\beta=2 / 3$. Therefore, substituting $x_{j} / A_{n}$ instead of $x$ in (3) and (4), then multiplying by $w_{j}$, and summing, we obtain

$$
\begin{gathered}
\frac{1}{A_{n}} \sum_{x_{j} \leq A_{n}}\left(w_{j} x_{j}-w_{j} A_{n}-\frac{w_{j} x_{j}\left(x_{j}-A_{n}\right)^{2}}{x_{j}^{2}+m_{j}^{2 / 3} M_{j}^{1 / 3}}\right)= \\
=\frac{1}{A_{n}} \sum_{x_{j} \leq A_{n}}\left(w_{j} x_{j}-w_{j} A_{n}-\frac{w_{j}\left(x_{j}-A_{n}\right)^{2}}{x_{j}+x_{j}^{1 / 3} A_{n}^{2 / 3}}\right) \leq \\
\leq \sum_{x_{j} \leq A_{n}} w_{j} \log \frac{x_{j}}{A_{n}} \leq \frac{1}{A_{n}} \sum_{x_{j} \leq A_{n}}\left(w_{j} x_{j}-w_{j} A_{n}-\frac{w_{j}\left(x_{j}-A_{n}\right)^{2}}{x_{j}+A_{n}}\right)= \\
=\frac{1}{A_{n}} \sum_{x_{j} \leq A_{n}}\left(w_{j} x_{j}-w_{j} A_{n}-\frac{w_{j} x_{j}\left(x_{j}-A_{n}\right)^{2}}{x_{j}^{2}+m_{j}^{1 / 2} M_{j}^{1 / 2}}\right)
\end{gathered}
$$

and

$$
\begin{gathered}
\frac{1}{A_{n}} \sum_{x_{j}>A_{n}}\left(w_{j} x_{j}-w_{j} A_{n}-\frac{w_{j} x_{j}\left(x_{j}-A_{n}\right)^{2}}{x_{j}^{2}+m_{j}^{1 / 2} M_{j}^{1 / 2}}\right)= \\
=\frac{1}{A_{n}} \sum_{x_{j}>A_{n}}\left(w_{j} x_{j}-w_{j} A_{n}-\frac{w_{j}\left(x_{j}-A_{n}\right)^{2}}{x_{j}+A_{n}}\right)<\sum_{x_{j}>A_{n}} w_{j} \log \frac{x_{j}}{A_{n}}< \\
<\frac{1}{A_{n}} \sum_{x_{j}>A_{n}}\left(w_{j} x_{j}-w_{j} A_{n}-\frac{w_{j}\left(x_{j}-A_{n}\right)^{2}}{x_{j}+x_{j}^{1 / 3} A_{n}^{2 / 3}}\right)= \\
=\frac{1}{A_{n}} \sum_{x_{j}>A_{n}}\left(w_{j} x_{j}-w_{j} A_{n}-\frac{w_{j} x_{j}\left(x_{j}-A_{n}\right)^{2}}{x_{j}^{2}+m_{j}^{1 / 3} M_{j}^{2 / 3}}\right) .
\end{gathered}
$$

Further, utilizing inequalities $m_{j}^{2 / 3} M_{j}^{1 / 3} \leq m_{j}^{1 / 2} M_{j}^{1 / 2} \leq m_{j}^{1 / 3} M_{j}^{2 / 3}$, we get

$$
\begin{gathered}
\frac{1}{A_{n}} \sum_{x_{j} \leq A_{n}}\left(w_{j} x_{j}-w_{j} A_{n}-\frac{w_{j} x_{j}\left(x_{j}-A_{n}\right)^{2}}{x_{j}^{2}+m_{j}^{2 / 3} M_{j}^{1 / 3}}\right) \leq \sum_{x_{j} \leq A} w_{j} \log \frac{x_{j}}{A_{n}} \leq \\
\leq \frac{1}{A_{n}} \sum_{x_{j} \leq A_{n}}\left(w_{j} x_{j}-w_{j} A_{n}-\frac{w_{j} x_{j}\left(x_{j}-A_{n}\right)^{2}}{x_{j}^{2}+m_{j}^{1 / 3} M_{j}^{2 / 3}}\right)
\end{gathered}
$$


and

$$
\begin{gathered}
\frac{1}{A_{n}} \sum_{x_{j}>A_{n}}\left(w_{j} x_{j}-w_{j} A_{n}-\frac{w_{j} x_{j}\left(x_{j}-A_{n}\right)^{2}}{x_{j}^{2}+m_{j}^{2 / 3} M_{j}^{1 / 3}}\right)<\sum_{x_{j}>A_{n}} w_{j} \log \frac{x_{j}}{A_{n}}< \\
<\frac{1}{A_{n}} \sum_{x_{j}>A_{n}}\left(w_{j} x_{j}-w_{j} A_{n}-\frac{w_{j} x_{j}\left(x_{j}-A_{n}\right)^{2}}{x_{j}^{2}+m_{j}^{1 / 3} M_{j}^{2 / 3}}\right) .
\end{gathered}
$$

Combining this together, we obtain

$$
\frac{1}{A_{n}} \sum_{j=1}^{n} \frac{w_{j} x_{j}\left(x_{j}-A_{n}\right)^{2}}{x_{j}^{2}+m_{j}^{2 / 3} M_{j}^{1 / 3}} \leq \log A_{n}-\log G_{n} \leq \frac{1}{A_{n}} \sum_{j=1}^{n} \frac{w_{j} x_{j}\left(x_{j}-A_{n}\right)^{2}}{x_{j}^{2}+m_{j}^{1 / 3} M_{j}^{2 / 3}},
$$

as desired.

(Sufficiency) Let $s, t \in \mathbb{R}$ with $1<t<s+1$. Set

$$
x_{1}=\frac{s+1-t}{s}, x_{2}=t, x_{j}=1(j=3, \ldots, n) ;
$$

$w_{1}=\frac{s}{s+1+(n-2) t}, \quad w_{2}=\frac{1}{s+1+(n-2) t}, \quad w_{j}=\frac{t}{s+1+(n-2) t}$ $(j=3, \ldots, n)$. Now, the same computation as in the proof of Theorem 2 (see [2]), provides that $\alpha \leq 1 / 3$ and $\beta \geq 2 / 3$.

Remark. Putting $x_{j}=\frac{q_{j}}{p_{j}}, w_{j}=p_{j} / \sum_{j=1}^{n} q_{j} \quad(j=1, \ldots, n)$, where $\sum_{j=1}^{n} p_{j}=\sum_{j=1}^{n} q_{j}$, in (5), we get (2).

By using Theorem 3, we obtain the following consequence.

Theorem 4. The inequality

$$
A_{n}-G_{n} \leq \sum_{j=1}^{n} \frac{w_{j} x_{j}\left(x_{j}-A_{n}\right)^{2}}{x_{j}^{2}+m_{j}^{\beta} M_{j}^{1-\beta}}
$$

holds for $\beta \geq 2 / 3$.

Proof. By the mean value theorem and Theorem 3, we have

$$
\begin{gathered}
A_{n}-G_{n}=\exp \left(\log A_{n}\right)-\exp \left(\log G_{n}\right) \leq A_{n}\left(\log A_{n}-\log G_{n}\right) \leq \\
\leq \sum_{j=1}^{n} \frac{w_{j} x_{j}\left(x_{j}-A_{n}\right)^{2}}{x_{j}^{2}+m_{j}^{\beta} M_{j}^{1-\beta}},
\end{gathered}
$$


which completes the proof.

Our next result refines sign of the first inequality in (5) for $\alpha=0$.

Theorem 5. If $C=\frac{1}{A_{n}} \sum_{j=1}^{n} \frac{w_{j} x_{j}\left(x_{j}-A_{n}\right)^{2}}{x_{j}^{2}+M_{j}}$, then

$$
C \leq C+s_{2} \leq C+s_{3} \leq \cdots \leq C+s_{n-1} \leq \log \left(A_{n}\right)-\log \left(G_{n}\right)
$$

Equality occurs if and only if all $x_{j}$ are equal.

Proof. By Lemma 3 we have

$$
C \leq C+s_{2} \leq C+s_{3} \leq \cdots \leq C+s_{n-1} .
$$

Now, we have to prove the last inequality in (7). Let's choose an arbitrary $x_{\mu_{j}} \in\left\{x_{1}, \ldots, x_{n}\right\}, 1 \leq \mu_{1}<\mu_{2}<\cdots<\mu_{n-1} \leq n$, with the corresponding weights $w_{\mu_{j}} \in\left\{w_{1}, \ldots, w_{n}\right\}$, and let $x_{\mu_{n}}=\left\{x_{1}, \ldots, x_{n}\right\} \backslash$ $\left\{x_{\mu_{1}}, \ldots, x_{\mu_{n-1}}\right\}$. Now, utilizing the first inequality in (5) with $\alpha=0$, we obtain

$$
\begin{gathered}
\log \left(A_{n}\right)=\log \left(w_{\mu_{n}} x_{\mu_{n}}+\left(\sum_{j=1}^{n-1} w_{\mu_{j}}\right) \frac{\sum_{j=1}^{n-1} w_{\mu_{j}} x_{\mu_{j}}}{\sum_{j=1}^{n-1} w_{\mu_{j}}}\right) \geq \\
\geq \frac{1}{A_{n}} \frac{w_{\mu_{n}} x_{\mu_{n}}\left(x_{\mu_{n}}-A_{n}\right)^{2}}{x_{\mu_{n}}^{2}+\max \left(x_{\mu_{n}}^{2}, A_{n}^{2}\right)}+ \\
+\frac{1}{A_{n}} \frac{\left(\sum_{j=1}^{n-1} w_{\mu_{j}}\right) \frac{\sum_{j=1}^{n-1} w_{\mu_{j}} x_{\mu_{j}}}{\sum_{j=1}^{n-1} w_{\mu_{j}}}\left(\frac{\sum_{j=1}^{n-1} w_{\mu_{j}} x_{\mu_{j}}}{\sum_{j=1}^{n-1} w_{\mu_{j}}}-A_{n}\right)^{2}}{\left(\frac{\sum_{j=1}^{n-1} w_{\mu_{j}} x_{\mu_{j}}}{\sum_{j=1}^{n-1} w_{\mu_{j}}}\right)^{2}+\max \left(\left(\frac{\sum_{j=1}^{n-1} w_{\mu_{j}} x_{\mu_{j}}}{\sum_{j=1}^{n-1} w_{\mu_{j}}}\right)^{2}, A_{n}^{2}\right)}+ \\
+\frac{1}{\log \left(x_{\mu_{n}}^{w_{\mu_{n}}}\left(\frac{\sum_{j=1}^{n-1} w_{\mu_{j}} x_{\mu_{j}}}{\sum_{j=1}^{n-1} w_{\mu_{j}}}\right)^{\sum_{j=1}^{n-1} w_{\mu_{j}}}\right)}= \\
\sum_{j=1}^{n} \frac{w_{j} x_{j}\left(x_{j}-A_{n}\right)^{2}}{x_{j}^{2}+\max \left(x_{j}^{2}, A_{n}^{2}\right)}-\frac{1}{A_{n}} \sum_{j=1}^{n-1} \frac{w_{\mu_{j}} x_{\mu_{j}}\left(x_{\mu_{j}}-A_{n}\right)^{2}}{x_{\mu_{j}}^{2}+\max \left(x_{\mu_{j}}^{2}, A_{n}^{2}\right)}+
\end{gathered}
$$




$$
\begin{gathered}
+\log \left(G_{n}\right)-\sum_{j=1}^{n-1} w_{\mu_{j}} \log x_{\mu_{j}}+\left(\sum_{j=1}^{n-1} w_{\mu_{j}}\right) f_{A_{n}}\left(\frac{\sum_{j=1}^{n-1} w_{\mu_{j}} x_{\mu_{j}}}{\sum_{j=1}^{n-1} w_{\mu_{j}}}\right)= \\
=\log \left(G_{n}\right)+C+\left(\sum_{j=1}^{n-1} w_{\mu_{j}}\right) f_{A_{n}}\left(\frac{\sum_{j=1}^{n-1} w_{\mu_{j}} x_{\mu_{j}}}{\sum_{j=1}^{n-1} w_{\mu_{j}}}\right)-\sum_{j=1}^{n-1} w_{\mu_{j}} f_{A_{n}}\left(x_{\mu_{j}}\right) .
\end{gathered}
$$

Since $x_{\mu_{j}}, i=\{1, \ldots, k\}$ are arbitrary, the last inequality in (7) holds. The theorem is proved.

Putting $x_{j}=\frac{q_{j}}{p_{j}}, w_{j}=p_{j} / \sum_{j=1}^{n} q_{j}(j=1, \ldots, n)$, where $\sum_{j=1}^{n} p_{j}=$ $=\sum_{j=1}^{n} q_{j}$, in Theorem 5 , we obtain the following refinement of the first inequality in (1).

Corollary 1. Let $p_{j}, q_{j}(j=1, \ldots, n)$ be positive real numbers satisfying $\sum_{j=1}^{n} p_{j}=\sum_{j=1}^{n} q_{j}$. Then

$\sum_{j=1}^{n} \frac{q_{j}\left(q_{j}-p_{j}\right)^{2}}{q_{j}^{2}+\hat{M}_{j}}=\hat{C} \leq \hat{C}+\hat{s}_{2} \leq \hat{C}+\hat{s}_{3} \leq \cdots \leq \hat{C}+\hat{s}_{n-1} \leq \sum_{j=1}^{n} p_{j} \log \frac{p_{j}}{q_{j}}$

where

$\hat{s}_{k}=\max _{1 \leq \mu_{1}<\mu_{2}<\cdots<\mu_{k} \leq n}\left[\left(\sum_{j=1}^{k} p_{\mu_{j}}\right) f_{1}\left(\frac{\sum_{j=1}^{k} q_{\mu_{j}}}{\sum_{j=1}^{k} q_{\mu_{j}}}\right)-\sum_{j=1}^{k} p_{\mu_{j}} f_{1}\left(\frac{q_{\mu_{j}}}{p_{\mu_{j}}}\right)\right]$, and $\hat{M}_{j}=\max \left(p_{j}^{2}, q_{j}^{2}\right) \quad(j=1, \ldots, n)$.

Acknowledgment. The authors wish to express their thanks to Professor Mario Krnić for his valuable suggestions. This work was supported by the Asia Research Center at the National University of Mongolia and the Korea Foundation of Advanced Studies (Project No. 18, 2016-2017).

\section{References}

[1] Adiyasuren V., Batbold Ts., Adil Khan M. Refined arithmetic-geometric mean inequality and new entropy upper bound. Commun. Korean Math. Soc., 2016, vol. 31, no. 1, pp. 95-100. DOI: 10.4134/CKMS.2016.31.1.095

[2] Alzer H. On an inequality from information theory. Rend. Istit. Mat. Univ. Trieste, 2012, vol. 46, pp. 231-235. 
[3] Beckenbach E. F., Bellman R. Inequalities. Springer Verlag, Berlin, 1983.

[4] Bullen P. S., Mitrinović D. S., Vasić P. M. Means and their inequalities. Reidel, Dordrecht, 1988.

[5] Gao P. A new approach to Ky Fan-type inequalities. Int. J. Math. Math. Sci., 2005, no. 22, pp. 3551-3574. DOI: 10.1155/IJMMS.2005.3551

[6] Halliwell G. T., Mercer P. R. A refinement of an inequality from information theory. J. Inequal. Pure Appl. Math., 2004, vol. 5, no. 1, pp. 1-3.

[7] Hardy G. H., Littlewood J. E., Pólya G. Inequalities. Cambridge University Press, Cambridge, 1954.

[8] Mitrinović D. S. Analytic inequalities. Springer Verlag, New York, 1970.

[9] Parkash O., Kakkar P. Entropy bounds using arithmetic-geometricharmonic mean inequality. Int. J. Pure Appl. Math., 2013, vol. 89, no. 5, pp. 719-730. DOI: 10.12732/ijpam.v89i5.8

[10] Simic S. Jensen's inequality and new entropy bounds. Appl. Math. Lett., 2009, vol. 22, pp. 1262-1265. DOI: 10.1016/j.aml.2009.01.040

[11] Tian J. New property of a generalized Hölder's inequality and its applications. Information Sciences, 2014, vol. 288, pp. 45-54. DOI: 10.1016/j.ins.2014.07.053

Received October 16, 2016.

In revised form, January 30, $201 \%$.

Accepted January 30, $201 \%$.

Published online April 5, $201 \%$.

National University of Mongolia

P.O. Box 46A/104, Ulaanbaatar 14201, Mongolia

E-mail: V_Adiyasuren@yahoo.com, tsbatbold@hotmail.com 